theoretical possibility, laboratory evidence is elusive. Later, it was suggested that smallscale turbulence within a much larger current sheet would accelerate the reconnection rate due to global wandering of the magnetic field lines and emergence of multiple reconnection sites ${ }^{10}$; and even that smallscale turbulence, if ubiquitous, would break the frozen field condition everywhere ${ }^{11}$.

Recently, it was shown that an instability associated with reconnecting current sheets could cause the sheet to break up into a large number of plasmoid structures, broadening the reconnection region and increasing the rate of reconnection ${ }^{12}$. This led to an alternative model for turbulence within a current sheet. The plasmoids, which contain magnetic islands, seem to be particularly efficient at accelerating particles to high energies, due to the tendency of closed magnetic islands to shrink. It turns out that multiple magnetic islands can produce a power-law spectrum of accelerated particles, similar to the one often seen in natural plasmas ${ }^{13}$.
The formation of current sheets and filaments in magnetic turbulence, similar to the setting investigated by Retinò et al. ${ }^{4}$ has also received wide attention. Methods have been developed for identifying current sheets in numerical simulations of turbulence, and it has been shown that, despite occupying a miniscule fraction of the volume, they are responsible for a large fraction of the energy dissipated in a turbulent flow ${ }^{14}$.

The future of magnetic reconnection research is bright. The likely identification of reconnection as the origin of the highly relativistic particles responsible for gamma-ray flares in the Crab Nebula has demonstrated the importance of laboratory and Solar System reconnection studies spanning an even greater range of theoretical, computational and experimental research as illustrated in Fig. 1. On intermediate scales, NASA's Magnetospheric MultiScale mission has been launched to follow the Cluster mission - promising an even closer look at collisionless reconnection in the terrestrial magnetosphere.
Ellen Zweibel is in the Departments of Astronomy and Physics, University of Wisconsin, Madison, Wisconsin 53706-1507, USA.

e-mail:zweibel@astro.wisc.edu

References

1. Zweibel, E. G. \& Yamada, M. Ann. Rev. Astron. Astrophys. 47, 291-332 (2009).

2. Yamada, M., Kulsrud, R. \& Ji, H. Rev. Mod. Phys. 82, 603-664 (2010).

3. Xiao, C. J. et al. Nature Phys. 2, 478-483 (2006).

4. Retinò, A. et al. Nature Phys. 3, 235-238 (2007).

. Parker, E. N. J. Geophys. Res. 62, 509-520 (1957)

6. Furth, H. P., Killeen, J. \& Rosenbluth, M. N. Phys. Fluids 6, 459-484 (1963).

Greene, J. M. J. Geophys. Res. 93, 8583-8590 (1988).

8. Priest, E. R., Hornig, G. \& Pontin, D. I. J. Geophys Res. 108, 1285 (2003).

9. Eriksson, E. et al. Geophys. Res. Lett. 42, 6883-6889 (2015).

10. Lazarian, A. \& Vishniac, E. M. Astrophys. J. 517, 700-718 (1999).

11. Eyink, G. L., Lazarian, A. \& Vishniac, E. T. Astrophys. J. 743, 51-79 (2011)

12. Loureiro, N. F. \& Uzdensky, D. A. Plasma Phys. Contr. F. (in the press).

13. Drake, J. F., Swisdak, M. \& Fermo, R. Astrophys J. Lett. 763, 5-10 (2013).

14. Zhdankin, V., Uzdensky, D. A. \& Boldyrev, S. Phys. Rev. Lett. 114, 065002 (2015).

15. Den Hartog, D. J. \& Craig, D. Plasma Phys. Contr. F. 42, L47-L53 (2000).

16. Balbo, M., Walter, R., Ferrigno, C. \& Bordas, P. Astron. Astrophys. 527, L4 (2011).

\title{
STATISTICAL PHYSICS
}

\section{0 years of the Ising model}

Ernst Ising's analysis of the one-dimensional variant of his eponymous model (Z. Phys 31, 253-258; 1925) is an unusual paper in the history of early twentiethcentury physics. Its central result demonstrating that a linear chain of two-state spins cannot undergo a phase transition at finite temperature - is correct, if somewhat trivial compared with other physics breakthroughs published in the 1920s. But it is Ising's fateful extension of his conclusions to two and three dimensions that proved spectacularly wrong and, paradoxically, earned him an enduring association with the model that now bears his name.

A possible reason for Ising's unexpected celebrity is that his erroneous conclusions betray a superficial understanding of what turned out to be some of the deepest and far-reaching problems to be addressed in twentieth-century physics. The Hamiltonian of the model is simple to write down - it describes a network of spins interacting with each other through a coupling that only applies if the spins are next to each other but the physics it displays is rich and nontrivial: not only does it provide an intuitive device for illustrating the essential features of phase transitions and critical phenomena, it neatly encapsulates the main traits of

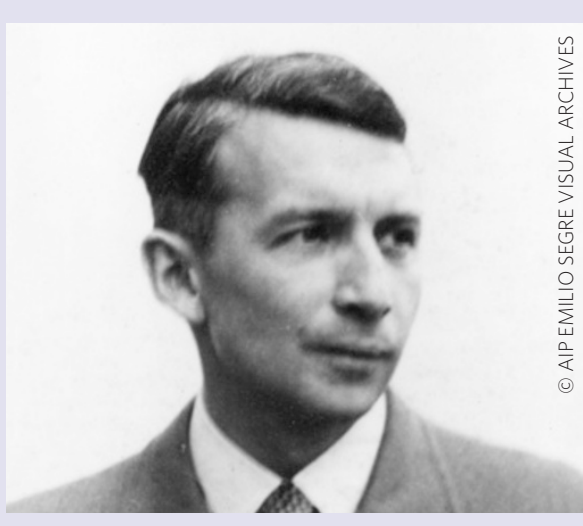

the many-body problem that has come to dominate areas such as condensed-matter physics. The broader class of spin models it belongs to was used to uncover concepts such as universality, renormalization, symmetrybreaking and emergence. Ising can perhaps be forgiven for not predicting all of that.

Famously, the two-dimensional version for the model was solved analytically by Lars Onsager in the early 1940s (Phys. Rev. 65, 117; 1944), a result that is rightly considered a towering achievement among many significant contributions made over the years by the likes of Peierls, Bethe, Yang, Kadanoff (see page 995) Fisher and Wilson, just to name a handful. But the three-dimensional lattice has never been solved exactly, in spite of a multitude of attempts and false dawns - including a claim by John Maddox (who would later become the editor of Nature) made at a conference in Paris in 1952.

Although the 3D model is thought by some to be analytically intractable (and has also been claimed to belong to the NP-complete category of computational decision problems), progress has continued and recent numerical techniques based on conformal field theory have shed further light on the structure of the problem (J. Stat. Phys. 157, 869-914; 2014). Nevertheless, the real value of the Ising model and its many derivatives lies precisely in the complexity they encapsulate. These have found use in fields as disparate as condensed-matter physics, physical chemistry, neuroscience and, more broadly, the study of so-called complex systems.

Ising studied a deceptively simple model that, unknown to him at the time, captures the essential physics of an extremely wide category of problems. He may have been wrong in his 1925 paper, but he tripped over a veritable physics goldmine.

ANDREA TARONI 